\title{
Percepción de los hombres ante la aceptación 0 ReChazo de la vasectomía. Cartagena 2013
}

\section{Perception of Men to the acceptance or Rejection OF the vasectomy. Cartagena 2013}

\section{Título corto: Percepción de los hombres ante la VASectomía}

Shirley Fernández Aragón ${ }^{1}$, Keydis Ruydiaz Gómez², Gloria Baza Maestre ${ }^{3}$, Dayelis Berrio Ayala ${ }^{3}$ y Yoel Rosales Barrios ${ }^{3}$

Recibido en diciembre 9 de 2013

Aceptado en marzo 26 de 2014

\section{Resumen}

La vasectomía es un procedimiento complejo que afecta la vida sexual de los hombres. Ante esta práctica existe una serie de mitos con respecto a la pérdida de virilidad o vigor sexual entre aquellos varones que se atreven a realizarse dicha intervención quirúrgica. Objetivo: Determinar la percepción de los hombres ante la práctica de la vasectomía en tres barrios de Cartagena en el año 2013. Metodología: Estudio de tipo descriptivo, discriminado así: En la fase inicial, se identificaron las características sociodemográficas y el nivel de conocimiento de los 162 participantes, distribuidos por 54 en cada barrio; en la segunda fase, se utilizó una muestra de 30 participantes, discriminados en 10 por cada barrio. Se utilizó un instrumento de 22 preguntas cerradas con justificación que describían los mitos y creencias ante la vasectomía. Resultados: La vasectomía es más aceptada entre los hombres de edades de 25-35 años que muestran mayor interés a la hora de participar en el control de la natalidad; por el contrario, un bajo porcentaje entre hombres de 20-25 años estarían dispuestos a realizarse el procedimiento Conclusiones: Son pocos los hombres que tienen mitos y creencias acerca del procedimiento quirúrgico masculino de planificación familiar consistente en la vasectomía.

Palabras Clave: Aceptación, hombres, percepción, planificación familiar vasectomía (DeCS).

\section{Abstract}

Vasectomy is a complex process that affects the sexual life of men, this practice there are a number of myths regarding the loss of virility or sexual force those men who dare to be performed this surgery. Objectives: To determine perception of men to the practice of vasectomy in 3 districts of Cartagena in 2013. Methodology:

\footnotetext{
1. Enfermera. Especialista en Gestión de la Calidad y Auditoría en Salud. Estudiante de Maestría en Enfermería. Integrante del Grupo GISIBEC del Programa de Enfermería de la CURN. Docente Programa de Enfermería de la CURN. Correo: shirley.fernandez@curnvirtual.edu.co

2. Enfermera, candidata a maestria en enfermeria con Enfasis en cuidado a las personas y colectivos, Docente de la Corporacion Universitaria Rafael Nuñez; Integrante del grupo GISIBEC. Keydiruidiazg@hotmail.com

3. Enfermera. Corporación Universitaria Rafael Núñez. Correos: gbazam06@curnvirtual.edu.co ; dberrioa06@curnvirtual.edu.co y yrosalesb06@curnvirtual.edu.co
} 
Descriptive, discriminated, the initial phase were identified sociodemographic characteristics and knowledge level of the 162 participants, divided 54 participants in each neighborhood; second phase where we used a sample of 30 participants discriminated by 10 for each neighborhood, using an instrument of 22 closed and open questions describing the myths and beliefs before the vasectomy. Results: Vasectomy is more accepted among men aged 25-35 years showing greater interest when participating in birth control, however a small percentage of men aged 20-25 would be willing to be held the procedure. Conclusions: There are few men who have myths and beliefs about the surgical procedure male family planning vasectomy

Keyword: Acceptance, men, perception, family planning vasectomy (MeSH).

\section{Introducción}

$\mathrm{E}$ n los programas de planificación familiar y en el control de la natalidad se deben identificar previamente todos los mitos y creencias que generan, en este caso el procedimiento quirúrgico masculino vasectomía, para constatar que los hombres gocen de una buena salud reproductiva. La salud reproductiva es "un estado general de bienestar físico, mental y social, y no es simplemente la ausencia de enfermedades o dolencias".

Actualmente, la vasectomía es considerada un procedimiento complejo que afecta la vida sexual de los hombres debido a la falta de información, lo que implica la poca utilización de los métodos de anticoncepción en la población masculina. Se estima que el costo de la vasectomía es aproximadamente cinco veces menor al costo de la esterilización tubárica ${ }^{1,2}$

En torno a la práctica de la vasectomía, aún existe una serie de mitos y prejuicios con respecto a una supuesta pérdida de virilidad o vigor sexual entre aquellos varones que se atrevan a realizarse dicha intervención quirúrgica menor ${ }^{3}$. Además, existen varias dudas sobre este método, lo cual se ha visto reflejado significativamente en la poca participación de los hombres en el mismo; son muchos los hombres que tienen diferentes puntos de vista acerca de la realización de la vasectomía, así como mitos y creencias que dificultan que se lleve a cabo este procedimiento. Esto podría generar que la población masculina tenga poca responsabilidad a la hora de escoger un método anticonceptivo que sea beneficioso para ella y sus parejas, teniendo en cuenta que la vasectomía es un procedimiento simple, que involucra menos riesgos que la ligadura de trompas ${ }^{4}$.
"El mito más común se refiere al desempeño sexual, no existe la posibilidad de que se presenten problemas con la erección peneana debido a una vasectomía. El mito machista que asocia la impotencia a la vasectomía expresa el miedo de que la masculinidad esté vinculada a los órganos genitales y que cualquier intervención sobre ellos pueda perjudicar el desempeño sexual"5.

Así, surge la necesidad de indagar desde el campo de la enfermería el impacto que genera la vasectomía en los hombres. El enfoque y los resultados de la investigación permitieron soportar y fundamentar las prácticas asistenciales, investigativas y académicas desde el pilar de la salud sexual y reproductiva, forjando así las competencias del profesional de enfermería en el contexto cultural y brindando bases para abordar a la población desde la educación en planificación familiar masculina, con el fin de determinar la percepción de los hombres ante la vasectomía sobre los mitos y creencias, por medio de la identificación de sus características demográficas, nivel de conocimiento y descripción de sus mitos y creencias que influyen para practicarse el procedimiento.

\section{Materiales y Métodos}

Estudio de naturaleza cuantitativa de tipo descriptivo transversal, el cual se limita a observar y describir la frecuencia con la que se presentan las variables elegidas ${ }^{6}$. Fue realizado en dos fases: Una fase inicial en la cual se identificaron las características sociodemográficas y el nivel de conocimiento de los 162 participantes, discriminados en 54 participantes en cada barrio (Olaya, Blas de Lezo y El Recreo); y una segunda fase en la cual se utilizó una muestra de 30 participantes, discriminados en 10 por cada barrio, los cuales debían 
cumplir los siguientes criterios: ser hombres con rangos de edades entre 20 y 49 años, que residieran en los barrios escogidos, que tuvieran o no vida sexual activa, que aceptaran participar en el estudio, y que no tuvieran discapacidad cognitiva. Se aplicó un instrumento de 22 preguntas cerradas con respuestas justificadas que describían los mitos y creencias ante la vasectomía, instrumento al que se le realizó una validación facial para obtener los resultados. Los datos obtenidos en la investigación fueron digitados en hoja de cálculo Microsoft Excel y fueron procesados por el paquete estadístico SPSS 19 (Statistical Package for the Social Sciences) representados en tablas y gráficos. Se tuvo en cuenta que cada participante de la investigación firmara el consentimiento informado antes de suministrar la información, basado en los criterios éticos de la investigación estimados por la resolución 008430 de 1993.

\section{Resultados}

\section{Características sociodemográficas}

El rango de edad de los participantes oscila entre 20 a 49 años de edad. El estado civil predominante es el soltero en los tres barrios, estimado en un $60 \%$. El número de hijos varía de 1 a 4; todos los hombres que no tienen hijos desean tenerlos a futuro. El salario mínimo legal vigente (SMLV) está entre 1 y 3. Los estratos socioeconómicos de los barrios son 1, 3 y 4 . En cuanto al nivel educativo en el barrio El Recreo la mayoría tiene un alto nivel educativo, en el barrio Blas de Lezo oscilaban entre universitario y técnico, y en el barrio Olaya el nivel educativo predominante fue secundaria. Una cantidad representativa de los entrevistados ha tenido más de cinco compañeras sexuales y refiere tener una actualmente.

\section{Nivel de conocimiento}

Para la identificación del nivel de conocimiento sobre la vasectomía, se tuvo en cuenta la siguiente modalidad de calificación de la encuesta aplicada, hecha por los investigadores (Tabla 1).
Tabla 1. Modalidad de calificación por número de preguntas correctas.

\begin{tabular}{|l|c|c|}
\hline $\begin{array}{c}\text { MODO DE } \\
\text { CALIFICACIÓN }\end{array}$ & PUNTAJE & $\begin{array}{c}\text { No. DE PREGUNTAS } \\
\text { CORRECTAS }\end{array}$ \\
\hline Deficiente & $0-1,99$ & $0-3$ \\
\hline Insuficiente & $2-2,99$ & 4 \\
\hline Aceptable & $3-3,99$ & $5-6$ \\
\hline Excelente & $04-m a y$ & $07-a g o$ \\
\hline
\end{tabular}

Fuente: Encuesta sobre percepción de los hombres ante aceptación o rechazo de la vasectomía en tres barrios de Cartagena, 2013.

\section{Barrio Olaya}

El rango de edad entre 25 y 35 años posee un grado de conocimiento aceptable con un promedio de $3,43 \%$ conformado por 24 hombres que equivalen a un $50 \%$ de la muestra en estas edades, el $50 \%$ de la muestra restante de este rango de edad se ubica por debajo de las cinco preguntas correctas, teniendo el $29.16 \%$ un grado de conocimiento deficiente. Por otro lado, el grupo de jóvenes entre las edades de 20 y 25 años posee un grado de conocimiento aceptable, correspondiente al $38,46 \%$ de la muestra en este rango de edad, el 61,53 $\%$ (seis hombres) se encuentra por debajo de las cinco preguntas correctas, obteniendo el 30,76\% un grado de conocimiento deficiente. Así mismo, los hombres en edades entre 35 y 49 años presentaron un nivel de conocimiento aceptable estimado en un 35,29\%, el $23,52 \%$ presenta un grado de conocimientos deficientes, el $17,64 \%$ un nivel de conocimiento insuficiente y el $23,52 \%$ obtuvo un nivel de conocimiento excelente ante la vasectomía.

\section{Barrio Blas de Lezo}

El grado de conocimiento entre los rangos de edad de 20 - 25 y 25 - 35 años es excelente, con una calificación de 4,69, siendo el rango de 20 a 25 el de mayor conocimiento, equivalente al $42,85 \%$ de la muestra; el $57,14 \%$ se ubica en un grado de conocimiento aceptable. Por otro lado, los hombres entre las edades de 35 a 49 
años poseen un nivel de conocimiento aceptable, con un puntaje de 3,44 , equivalente a $29,41 \%$ de la muestra.

\section{Barrio El Recreo}

El grado de conocimiento entre los rangos de edad de 20 - 25 es excelente con un promedio de 4,38, siendo el rango de 25 a 35 el de mayor conocimiento, estimado en un $68,75 \%$ de la muestra en este rango de edad; el $31,25 \%$ se ubica en un grado de conocimiento aceptable, al igual que el grupo entre 35 y 49 años con un puntaje de 3,75 \%.

Al comparar los resultados obtenidos en los diferentes barrios, los rangos de edades entre 25-35 años obtuvieron el mejor puntaje, lo que evidencia que los hombres de esta edad poseen mayor información acerca del procedimiento vasectomía, situación que los aleja de los mitos y las creencias comunes, brindando las herramientas que permitan su participación activa en la planificación familiar y un mejor control de la natalidad, liberando a la mujer de ser el ente principal de la planificación familiar (Tabla 2).

Tabla 2. Comparación de puntajes según grado de conocimiento y rangos de edad

\begin{tabular}{|c|c|c|c|}
\hline \multirow{2}{*}{ RANGOS DE EDAD } & \multicolumn{3}{|c|}{ BARRIOS } \\
\cline { 2 - 4 } & EL RECREO & BLAS DE LEZO & OLAYA HERRERA \\
\hline $20-25$ & 4,38 & 4,69 & 3,43 \\
\hline $25-35$ & 4,69 & 4,69 & 3,43 \\
\hline $35-49$ & 3,75 & 3,44 & 3,13 \\
\hline
\end{tabular}

Fuente: Encuesta sobre percepción de los hombres ante aceptación o rechazo de la vasectomía en tres barrios de Cartagena, 2013.

\section{Mitos y creencias}

De acuerdo con el resultado obtenido, según los mitos y creencias sobre la vasectomía, se evidenció que ante la creencia de la pérdida del placer propio o de la pareja por realizarse dicha intervención, el 7 \% manifiesta que este procedimiento afecta su placer y el de la pareja. Por el contrario, el $100 \%$ de los encuestados expresa que la vasectomía no produce impotencia sexual; el $3 \%$ de la muestra estima que este procedimiento produce eyaculación precoz; mientras que el cambio de color y disminución del semen solo fue manifestado por el 10 $\%$ de la muestra.

Es relevante el porcentaje (43\%) ante los miedos para realizarse el procedimiento quirúrgico; la vergüenza representada solo en un $10 \%$, así como la disminución del apetito sexual representado en $6 \%$, mientras que el $100 \%$ estima que la vasectomía no produce impotencia sexual y el $10 \%$ manifiesta que el procedimiento no es seguro y puede atentar contra su salud. El 77 \% contestó que su esposa no debe cuidarse si su pareja se realiza la vasectomía; solo el $3 \%$ cree que la vasectomía lo protege de enfermedades de transmisión sexual; el $33 \%$ cree que el semen queda inmediatamente sin espermatozoides luego de realizada la cirugía; y un gran porcentaje de los hombres entrevistados (63\%) estaría dispuesto a realizarse la operación, datos que se relacionan con el nivel de educación y el rango de edad del grupo en estudio, así como la indagación de ellos ante el tema. En la Tabla 3, se evidencian los mitos y creencias más predominantes de acuerdo con las respuestas de los participantes. 
Tabla 3. Datos obtenidos acerca de los mitos y creencias de los mitos ante aceptación o rechazo de la vasectomía

\begin{tabular}{|c|c|c|c|c|}
\hline \multirow{2}{*}{ MITOS Y CREENCIAS } & \multicolumn{2}{|c|}{ SI } & \multicolumn{2}{|c|}{ NO } \\
\hline & FRECUENCIA & PORCENTAJE & FRECUENCIA & PORCENTAJE \\
\hline Afecta el placer propio o de su pareja & 2 & $7 \%$ & 28 & $93 \%$ \\
\hline Produce Impotencia sexual & 0 & $0 \%$ & 30 & $100 \%$ \\
\hline Produce eyaculación precoz & 1 & $3 \%$ & 29 & $97 \%$ \\
\hline Cambia el color de semen & 3 & $10 \%$ & 27 & $90 \%$ \\
\hline Siente miedo al realizarse & 13 & $43 \%$ & 17 & $57 \%$ \\
\hline Produce vergüenza & 3 & $10 \%$ & 27 & $90 \%$ \\
\hline Disminuye apetito sexual & 2 & $7 \%$ & 28 & $93 \%$ \\
\hline Es inseguro y atentaría contra su salud & 3 & $10 \%$ & 27 & $90 \%$ \\
\hline Su esposa también debe cuidarse & 7 & $23 \%$ & 23 & $77 \%$ \\
\hline Protege de ETS & 2 & $3 \%$ & 28 & $97 \%$ \\
\hline $\begin{array}{l}\text { Semen queda inmediatamente sin } \\
\text { espermatozoides }\end{array}$ & 10 & $33 \%$ & 20 & $67 \%$ \\
\hline Se realizaría la vasectomía & 19 & $63 \%$ & 11 & $37 \%$ \\
\hline TOTAL PARTICIPANTES & & & 30 & \\
\hline
\end{tabular}

Fuente: Encuesta sobre percepción de los hombres ante aceptación o rechazo de la vasectomía en tres barrios de Cartagena, 2013.

\section{Perdida del placer sexual}

El placer sexual es el goce o disfrute sexual, satisfacción, sensación agradable producida por la realización de actos sexuales ${ }^{[5]}$. Partiendo de las respuestas de los participantes, se evidenció que estos perciben la vasectomía como un método de planificación familiar confiable, que no atentaría contra su placer sexual ni el de su pareja. Aun desconociendo las causas por las que se produce el placer sexual en una persona, consideran que el mismo no está ligado al corte de los conductos deferentes, demostrado por respuestas como:

- Código 4: "Pienso que no, porque es un procedimiento interno que consiste en desconectar la parte que produce los espermatozoides con el líquido eyaculador".

- Código 9: "No creo que haciéndome la vasectomía pueda influir en las relaciones de mi pareja, porque pienso que le daría más seguridad a la pareja para tener relaciones".

\section{Impotencia sexual}

Partiendo de la información suministrada, se infiere que los hombres desvinculan absolutamente la impotencia sexual de la realización de la vasectomía, teniendo en cuenta que consideran que la erección está dada por el sistema nervioso, por hormonas, y, por otro lado, que la disfuncionalidad masculina está controlada por la parte psicológica.

- Código 23: "No, porque al cortar el conducto deferente no creo que eso influye en lo que manda la información que es el cerebro".

- Código 16: "No, pues eso tiene que ver mucho con hormonas y estado físico, no creo que cause impotencia sexual”.

\section{Miedo y temor}

Las respuestas de los participantes están parcialmente divididas, mientras que algunos no temen realizarse 
el procedimiento y lo aceptan como método de planificación, otros temen a la realización de este procedimiento por miedo a no poder revertirla, someterse al procedimiento invasivo, a no quedar iguales, y por temor a los posibles efectos secundarios después de la cirugía; como vemos en las siguientes respuestas.

- Código 3: "Sinceramente sí sentiría miedo porque es un cuerpo extraño, me sentiría anormal porque uno viene normal".

- Código 13: "Lo que he leído me ha ilustrado y pienso que no debo tener miedo, pero he optado por que la señora se haga la ligadura de trompas de Falopio".

- Código 18: "No, no creo es un mito, pero la verdad es que tenemos que entrar ya por esa planificación”.

\section{Aceptación o rechazo ante la vasectomía}

Los participantes argumentan que se realizarían el procedimiento de la vasectomía sin temor debido a que de esta manera ayudarían a su pareja en el control de la natalidad, y permitiría disfrutar de las relaciones sexuales sin pensar en embarazos. Por el contrario, algunos manifiestan que no se realizarían el procedimiento por temor, no a la cirugía como tal, sino a cualquier procedimiento invasivo por muy sencillo que sea. De igual manera, hay quienes consideran no estar de acuerdo con el procedimiento ya que atribuyen la planificación familiar como a una práctica netamente de la población femenina.

- Código 8: "No me realizaría la vasectomía, planificar es para las mujeres y no es que sea machista".

- Código 4: "Llegado el caso, el momento me la realizaría, es un método de planificación sexual y familiar, todo va de acuerdo a la relación y comprensión que haya con la pareja”.

- Código 2: "La verdad es que sí, si alguien llega y me comenta sobre el procedimiento me la realizaría, no me daría miedo, lo contrario".

\section{Discusión}

Los resultados de este estudio reportaron que las edades de los participantes oscilan entre 20 - 49 años, de nivel económico bajo y medio, y nivel educativo técnico y profesional. Hallazgos similares expresa Córdoba ${ }^{7}$ en su investigación, según la cual la edad de los pacientes que se realizaron la vasectomía estuvo en promedio de 36.6 años; es un dato importante para conocer las razón donde se concentra y se refleja la mayor preocupación por parte de los hombres por controlar su vida sexual reproductiva ${ }^{7}$. Al igual que Lara ${ }^{8}$ que también expresa el nivel socioeconómico: medio ( $25 \%$ ), muy alto ( $24.7 \%)$ y medio alto (20.1 \%), con una escolaridad secundaria.

En los hallazgos encontrados sobre los mitos y creencias, el 93,33\% manifiesta que no afecta el placer propio o de la pareja, al igual que la impotencia sexual y tampoco genera eyaculación precoz. Ante esto se evidencia que existe un grado de aceptación y conocimiento sobre el procedimiento; esto apoya lo manifestado en la investigación de Córdoba, Mercado y Sapiens, quienes encontraron que la vasectomía resultó ser un buen método para disfrutar e incrementar la frecuencia de relaciones sexuales, al dejar de preocuparse las parejas por embarazos no deseados ${ }^{1}$.

En contraste con lo anterior, en lo que concierne a la planificación familiar de acuerdo con los resultados se puede afirmar que los hombres consideran que la responsabilidad recae únicamente sobre su pareja. La explicación de esto se basa en algunas teorías ${ }^{9}$ que plantean que la sexualidad en los varones se comprende como un ejercicio de poder y la refieren a una masculinidad obsesiva, la cual expone como ideología una personificación del poder de los hombres sobre la mujer. Frente a lo anterior, y en concordancia con este trabajo, se ha encontrado que la presencia de los hombres en los hechos relacionados con la regulación de la fecundidad y la reproducción es poca, pues consideran que de esto se encarga la mujer, y rechazan generalmente el asumir responsabilidades en el ámbito reproductivo ${ }^{10}$. A pesar esto, y haciendo referencia específicamente a lo que representa la vasectomía, los hallazgos sugieren que la mayoría de hombres entrevistados tiene conocimientos aceptables respecto del procedimiento de la vasectomía, lo cual descarta, de cierta forma, que las ideas que puedan tener estén fundadas en mitos y creencias.

En este sentido, el resultado anterior puede tener fundamento en que precisamente la mayor parte de estos hombres son de edades relativamente jóvenes, y que hacen parte de un "cambio generacional" en el cual predominan ideas de mayor apertura en lo concerniente a la sexualidad y a que la mayoría de decisiones se toman en pareja ${ }^{10}$. Igualmente, existe evidencia similar sobre esta situación en los trabajos de Vega y Jaramillo³ , y de Cordoba, Mercado y Sapién ${ }^{[1]}$.

Es importante que el profesional de enfermería lidere procesos de enseñanza y educación en estas temáticas 
y no se presenten situaciones como las evidenciadas por Moura y $\mathrm{Col}^{11}$ en su investigación, en la cual "las enfermeras demostraron un mayor conocimiento acerca de los métodos naturales de anticoncepción y los ítems con mayor porcentaje de errores fueron los métodos de barrera. De igual forma, se encontró que el conocimiento de las enfermeras acerca de métodos anticonceptivos no era suficiente", necesitando más actualización y capacitación ${ }^{12}$.

Son pocos los hombres que tienen mitos y creencias acerca del procedimiento quirúrgico masculino de planificación familiar vasectomía, por lo que no se entiende la poca participación de estos en el control de la natalidad, atribuyendo la no implementación del método a miedos y temores a cualquier intervención quirúrgica por mínima que sea, lo cual impide que tomen esta importante decisión, olvidando por completo su responsabilidad en la planificación y sobrecargando la responsabilidad de las mujeres en el control de la natalidad; a su vez, el nivel educativo no es un factor que influya significativamente para que los hombres conozcan acerca de este método de planificación.

La sexualidad, tal como lo manifiestan los adolescentes y lo han dicho diferentes autores ${ }^{13,14,15}$, es considerada un tema tabú, por lo que se hace necesario poner este tema en la agenda pública. Los profesionales de enfermería deben enfatizar en la promoción de la vasectomía como un método de planificación familiar masculino quirúrgico, por lo que se da la necesidad de incorporar programas encaminados a educar a los hombres sobre la salud sexual y la importancia de su participación en la planificación familiar, con el objeto de despejar cualquier duda existente sobre este procedimiento.

A esto se suma que los programas de planificación familiar en nuestro contexto se centran exclusivamente en las mujeres, entendiéndose que estas disponen de más métodos anticonceptivos que los hombres. Por ello es importante fomentar la idea de que la fecundidad es un asunto de pareja, y que el sector donde habitan influye en las creencias y decisiones basadas en el contexto cultural, en cuanto a su reproducción sexual la planificación familiar permite establecer el número de hijos que desean tener, garantizando una mejor calidad de vida y el bienestar físico, psicológico, económico y social del nuevo $\operatorname{ser}^{19,20}$.

\section{Referencias Bibliográficas}

1. Córdoba Basulto DI, Mercado Sánchez T, Sapién López JS. Opiniones y experiencias de la vida sexual de mujeres con parejas con vasectomía. Rev. Esp. Med. Quir. [revista en la Internet]. 2010 [Citado 25 noviembre de 201]; 15(3):144-151. Disponible en: http://www. redalyc.org/articulo.oa? $\mathrm{id}=47316054008$

2. Untiveros Mayorga, Ch F, Mauricio Pachas J. Consecuencias a largo plazo de la vasectomía, en varones operados en el Hospital Nacional Cayetano Heredia. Rev. Med. Hered [revista en la Internet]. 2004 [Citado 25 noviembre de 201]; 15(3): 131-135. Disponible en: http://www.scielo.org.pe/scielo. php? script $=$ sci_arttext\&pid $=$ S1018-130X2004000300 $003 \& \operatorname{lng}=$ es\&nrm $=$ iso

3. Vega G, Jaramillo M. Percepciones y actitudes de los hombres de la frontera norte de México en relación con el uso de condones, práctica de la vasectomía y chequeos de próstata. Rev. Gerencia y Políticas de Salud. 2010; 9(18): 50-77.

4. Ministerio de Salud. Normas nacionales para la regulación de la fertilidad. [Internet]. Chile: MINSAL [citado 24 Abr. 2013]. Disponible en: http://web. minsal.cl/portal/url/item/795c63caff4ede9fe0400101 1f014bf2.pdf

5. Viveros M. La esterilización masculina: ¿Un punto de inflexión en las trayectorias anticonceptivas y reproductivas? Reflexiones a partir de un estudio de caso colombiano. Rev. Latinoamericana [revista en la Internet]. 2009 [citado 2013 Oct. 10]; (1):1129. Disponible en: http://www.e-publicacoes.uerj. br/index.php/SexualidadSaludySociedad/article/ download/3/9.

6. Hernández R, Fernández C, Baptista P. Metodología de la Investigación. México D.F.: Mc Graw-Hill Interamericana; 2010.

7. Córdoba D, Sapiens J, Valdepeña R, Patiño S, Castellanos H. Vasectomía: reseña de 25 años en el Centro de Cirugía Ambulatoria del ISSSTE. Rev. Esp. Méd. Quir. [revista en la Internet]. 2011 [citado 2013 Sep. 10]; 16(1):33-38. Disponible en: http:// www.medigraphic.com/pdfs/quirurgicas/rmq-2011/ rmq111g.pdf

8. Lara R, Velázquez N, Reyes E. Vasectomía sin bisturí. Perfil del usuario y resultados. Rev. Ginecol. Obstet. Mex. [revista en la Internet]. 2010 [citado 2013 Oct. 20]; 78(4):226-231. Disponible en: http:// www.medigraphic.com/pdfs/ginobsmex/gom-2010/ gom104d.pdf 
9. Kaufman M. 1987. The construction of masculinity and the triad of men's violence. En: Beyond Patriarchy: Essays by Men on Pleasure, Power, and Change. (M. Kaufman, ed.) pp. 1-29. Toronto; New York: Oxford University Press. Disponible en: http://jft-newspaper. aub.edu.lb/reserve/data/sp10127/sp10127.pdf

10. Figueroa J. Algunos elementos para interpretar la presencia de los varones en los procesos de salud reproductiva. Cad. Saúde Públ. [revista en la Internet]. 1998 [citado 2013 Oct 16]; 14(1): 87-96. Disponible en: http://www.scielo.br/pdf/csp/v14s1/1344.pdf

11. Moura E, Ferreira S, Costa M, De Sousa P, Americo C, Bezerra A. Conocimiento de enfermería acerca de los métodos anticonceptivos en el contexto del programa de salud de la familia. Rev. Enferm. Global [revista en la Internet]. 2010 [citado 2013 Nov. 12]; 20:1-10. Disponible en: http://revistas.um.es/eglobal/article/ viewFile/110911/105281

12. Vásquez M. Significado da regulaçao da fecundidade dos (as) adolescentes nma comunidade urbana marginal. Brasil: Universidad Federal de Santa Catarina; 2001.

13. Maldonado MC. Relatos de padres y madres sobre la educación sexual: Algunas reflexiones. Cali: Universidad del Valle; 2003.

14. Canaval G, Cerquera G, Hurtado N, Lozano J. Salud de los adolescentes y regulación de la fecundidad. Investigación y Educación en Enfermería [revista en la Internet]. 2006 [citado el 25 de noviembre 2013]; 24(1): 26-35. Disponible en: http://www.scielo.org.co/scielo. php?pid = S0120-53072006000100003\&script $=$ sci arttext\&tlng $=$ es
15. Silva C, Brêtas J, Ferreira D, Correa D, Cintra C. Uso da camisinha por adolescentes e jovens: avaliação as seqüência dos procedimentos. Acta Paulista de Enfermagem. 2010 Out/Dez; 14(4):392-9.

16. Instituto Geográfico Agustín Codazzi. Ubicación geográfica de barrios de Cartagena de Indias. [Internet]. Bogotá: IGAC [Citado 8 de abril de 2013]. Disponible en: http://geoportal.igac.gov.co/ssigl2.0/ visor/galeria.req? mapaId $=60$

17. Vidal A, Garzón K. Factores que influyen en la aceptabilidad del uso de la vasectomía como estrategia para la disminución de embarazos por parte de miembros de las fuerzas militares de Colombia. [Tesis de maestría]. Bogotá: Repositorio institucional EdocUR, Universidad del Rosario; 2013. [Citado 14 de marzo de 2014]. Disponible en: http://repository.urosario. edu.co/bitstream/handle/10336/4363/53161187-2013. pdf? sequence $=3$ \&isAllowed $=y$

18. Instituto de Urología y Medicina Sexual en Zaragoza. Reversión de la vasectomía. [internet]. España. [citado 5 Mar. 2012]. Disponible en: http://www. institutouroandrologico.com/servicios/reversion_ vasectomia.php

19. Figueroa J. Algunos elementos para interpretar la presencia de los varones en los procesos de salud reproductiva. Cad. Saúde Públ. [revista en la Internet]. 1998 [citado 17 Feb. 2014]; 14(suppl.1):87-96. Disponible en: http://www.scielo.br/scielo.php?pid = S0102311X1998000500018\&script $=$ sci_arttext

20. Berquó E, Cavenaghi S. Direitos reprodutivos de mulheres e homens face à nova legislação brasileira sobre esterilização voluntária. Cad. Saúde Públ. 2013;19(2):441-53.

Para citar este artículo:

Fernández S, Ruydiaz K, Baza G, Berrío D, Rosales Y. Percepción de los hombres ante la aceptación 0 rechazo de la vasectomía. Cartagena 2013. 2014 Jun; 11(1): 22 - 29 